\title{
ON THE DEMIREGULARITY OF WEAK SOLUTIONS OF NONLINEAR ELLIPTIC EQUATIONS
}

\author{
BY JINDŘICH NEČAS
}

Communicated by Felix Browder, June 29, 1970

1. Introduction. Let $\Omega$ be a bounded domain with infinitely differentiable boundary $\partial \Omega$ in $n$-dimensional real space $R_{n}$. Let $k$ be a positive integer, and let us define the functions $a_{i}(x, \xi)$ for multiindices $|i|=i_{1}+i_{2}+\cdots+i_{n} \leqq k$, continuous in $\bar{\Omega} \times R_{\kappa}$, where $\kappa$ is the number of indices of length $\leqq k$. By $W_{p}^{(k)}(\Omega)$, we denote the Sobolev space of $L_{p}$-functions whose derivatives up to the order $k$ are also $L_{p}$-functions, with the norm

$$
\|u\|_{k, p}=\left(\int_{\Omega|i| \leq k}\left|D^{i} u\right|^{p} d x\right)^{1 / p},
$$

where the usual notation

$$
D^{i}=\frac{\partial^{|i|}}{\partial x_{1}^{i_{1}} \cdots \partial x_{n}^{i_{n}}}
$$

is introduced. The functions $a_{i}(x, \xi)$ are supposed to satisfy the growth-conditions:

(1.1) $\left|a_{i}(x, \xi)\right| \leqq c(1+|\xi|)$.

Let functions $u_{0} \in W_{2}^{(k)}(\Omega)$ and $f_{i} \in L_{2}(\Omega),|i| \leqq k$, be given. Let $\dot{W}_{p}^{(k)}(\Omega)$ be the closure of $D(\Omega)$, the space of infinitely differentiable functions with compact support, in the space $W_{p}^{(k)}(\Omega)$.

A function $u$ from $W_{2}^{(k)}(\Omega)$ is called a weak solution of the Dirichlet problem: $\partial^{l} u / \partial n^{l}=\partial^{l} u_{0} / \partial n^{l}$ on $\partial \Omega, l=0,1, \cdots, k-1$, (where $\partial / \partial n$ is the derivative with respect to the outer normal),

$$
\sum_{|i| \leq k}(-1)^{|i|} D^{i}\left(a_{i}(x, \xi(u))\right)=\sum_{|i| \leqslant k}(-1)^{|i|} D^{i} f_{i} \quad \text { in } \Omega
$$

(where the components of $\xi(u)$ are $D^{j} u$ ) if

(1.2) $u-u_{0} \in \dot{W}_{2}^{(k)}(\Omega)$,

(1.3) for every $v$ in $\dot{W}_{2}^{(k)}(\Omega)$ :

$$
\int_{\Omega|i| \leq k} D^{i} v a_{i}(x, \xi(u)) d x=\int \sum_{\Omega|i| \leq k} D^{i} v f_{i} d x .
$$


We will suppose the following:

$$
\sum_{|i| \leq k} a_{i}(x, \xi) \xi_{i} \geqq c_{1} \sum_{|i| \leq k} \xi_{i}^{2}-c_{2} .
$$

For the sake of simplicity, we suppose the differentiability of $a_{i}(x, \xi)$ with respect to $\xi_{j}$ and

$$
\left|\frac{\partial a_{i}}{\partial \xi_{j}}\right| \leqq c, \quad \sum_{|i|,|j| \leq k} \frac{\partial a_{i}}{\partial \xi_{j}} \eta_{i} \eta_{j} \geqq c \sum_{|i|=k} \eta_{i}^{2} .
$$

The following condition for asymptotic behaviour of $a_{i}(x, \xi)$ is required: there exists continuous $a_{i j}(x)$ in $\bar{\Omega},|i|,|j| \leqq k$, such that

$$
\sum_{|i|,|j| \leq k} a_{i j}(x) \xi_{i} \xi_{j} \geqq c_{1} \sum_{|i|=k} \xi_{i}^{2}
$$

and such that for $t>0$ :

$$
\left|\frac{a_{i}(x, t \xi)}{t}-\sum_{|j| \leq k} a_{i j}(x) \xi_{j}\right| \leqq c(t)(1+|\xi|),
$$

where $c(t) \rightarrow 0$ for $t \rightarrow \infty$.

The main result is:

THEOREM. Let $2 \leqq p<\infty$ and $u_{0} \in W_{p}^{(k)}(\Omega), f_{i} \in L_{p}(\Omega)$. Let the conditions (1.1), (1.4)-(1.7) be satisfied. Then there exists a unique weak solution of the Dirichlet problem belonging to the space $W_{p}^{(k)}(\Omega)$. It satisfies the inequality:

$$
\|u\|_{k, p} \leqq c(p)\left(1+\sum_{|i| \leq k}\left\|f_{i}\right\|_{0, p}+\left\|u_{0}\right\|_{k, p}\right) .
$$

It is well known that the regularity problem consists of proving that the weak solution belongs to the class $C^{(k), \mu}$, the class of functions whose derivatives up to order $k$ are $\mu$-Hölder continuous (in $\Omega$ or $\bar{\Omega})$. The solution of this problem is not known in general. Under certain conditions, given more general growth of the functions $a_{i}(x, \xi):\left|a_{i}(x, \xi)\right| \leqq c\left(1+|\xi|^{m-1}\right), 1<m<\infty$, the answer is affirmative for the case of one second-order equation; see, for example, $O$. A. Ladyženskaja-N. N. Uralceva [5], Ch. B. Morrey [7], and for $n=2$, $k \geqq 1$, see J. Nečas [9]. For higher dimensions and order, or for systems of second or higher order, this problem is still open. There is a counterexample under a slightly different hypothesis for the secondorder systems of E. Giusti and M. Miranda [4], where the solution is bounded, but not continuous. This situation implies the definition of partial regularity: there exists a set $F$ closed in $\Omega$ with $\operatorname{mes}(F)=0$, such that the weak solution belongs to $C^{(k), \mu}(\Omega \backslash F)$. 
Partial regularity was proved in the papers of Ch. B. Morrey [8], E. Giusti-M. Miranda [3], E. Giusti [2]. If we look to the scale $W_{p}^{(k)}, 2 \leqq p \leqq \infty$, and if we extend it further to $C^{(k), \mu}$ for $0<\mu<1$, we see that the cut between weak and regular solutions is the space $W_{\infty}^{(k)}$. Hence, a weak solution is called demiregular if $u \in \bigcap_{p \geq 2} W_{p}^{(k)}(\Omega)$, and this is an immediate consequence of our theorem, provided $u_{0} \in W_{\infty}^{(k)}(\Omega)$ and $f_{i} \in L_{\infty}(\Omega)$.

2. Proof of the Theorem. We use the following nontrivial lemma from the theory of linear elliptic equations, see, for example, J. L. Lions, E. Magenes [6].

LEMMA 1. Let w be a weak solution of

$$
\sum_{|i|,|j| \leq k}(-1)^{|i|} D^{i}\left(a_{i j}(x) D^{j} w\right)=\sum_{|i| \leq k}(-1)^{|i|} D^{i} f_{i}
$$

in $\Omega$, with $f_{i} \in L_{p}(\Omega), \infty>p>1, w-u_{0} \in W_{p}^{(k)}(\Omega), u_{0} \in W_{p}^{(k)}(\Omega)$ and with $a_{i j}$ satisfying (1.6). Then there exists a unique solution and

$$
\|w\|_{k, p} \leqq\left(\sum_{|i| \leq k}\left\|f_{i}\right\|_{0, p}+\left\|u_{0}\right\|_{k, p}\right) .
$$

As an immediate consequence of Lemma 1, we obtain:

LEMma 2. For $w \in \dot{W}_{p}^{(k)}(\Omega), \infty>p \geqq 2$,

$$
\sup _{\|\bullet\| k, p^{\prime} \leq 1, v \in W_{p^{\prime}}(\Omega)} \int_{\Omega|i|,|j| \leq k} \sum_{i j}(x) D^{i}{ }_{v}^{j} D^{j} d x \geqq c(p)\|w\|_{k, p},
$$

where $1 / \mathrm{p}^{\prime}+1 / p=1$.

Using well-known results about monotone operators, their applications to nonlinear boundary value problems, compare F. E. Browder [1], we have:

Lemma 3. Under the conditions (1.1), (1.4), (1.5), there exists a unique solution of (1.2), (1.3) and

$$
\|u\|_{k, 2} \leqq c\left(1+\sum_{|i| \leq k}\left\|f_{i}\right\|_{0,2}+\left\|u_{0}\right\|_{k, 2}\right) .
$$

Proof of the theorem. Let $0 \leqq \tau \leqq 1$, and let us consider the family of differential operators defined as

$$
(1-\tau) \sum_{|i|,|j| \leq k}(-1)^{|i|} D^{i}\left(a_{i j}(x) D^{i} u\right)+\tau \sum_{|i| \leq k}(-1)^{|i|} D^{i}\left(a_{i}(x, \xi(u))\right) .
$$

We can easily see that the conditions (1.1), (1.4), (1.5) and (1.7) are valid with constants independent of $\tau$. Hence, for $0 \leqq \tau \leqq 1$, there 
exists a unique solution of our problem in $W_{2}^{(k)}(\Omega)$. For $\tau=0$, we have, in virtue of Lemma 1 , the assertion of the theorem.

(i) Let the assertion be valid for some $\tau_{0}$. Then it is true for $\tau_{0} \leqq \tau<\tau_{0}+\epsilon \leqq 1$ with some $\epsilon>0$.

Let $v \in W_{p}^{(k)}(\Omega)$ be such that $v-u_{0} \in \dot{W}_{q}^{(k)}(\Omega)$ and let us define the operator $A: v \rightarrow A v$ such that $A v$ is the solution of the problem with the functions

$$
f_{i}+\left(\tau-\tau_{0}\right) \sum_{|j| \leq k} a_{i j}(x) D^{j} v+\left(\tau_{0}-\tau\right) a_{i}(x, \xi(v))
$$

substituted for $f_{i}$.

For $\tau=\tau_{0}$, we obtain from (1.8) that the solution belongs to the ball $\|u\|_{k, p} \leqq R$ where

$$
R=(c(p)+1)\left(1+\sum_{|i| \leq k}\left\|f_{i}\right\|_{0, p}+\left\|u_{0}\right\|_{k, p}\right) .
$$

Let us take first $v$ in the ball $\|v\|_{k, p} \leqq 2 R$ and then $\epsilon$ small enough such that $\|A v\|_{k, p} \leqq 2 R$. It follows from (1.5):

$$
\begin{aligned}
\int \sum_{\Omega|i| \leq k}\left(a_{i}\left(x, \xi\left(u_{1}\right)\right)-a_{i}\left(x, \xi\left(u_{2}\right)\right)\right) D^{i}\left(u_{1}-u_{2}\right) d x & \\
& \geqq c \sum_{|i| \leq k}\left\|D^{i}\left(u_{1}-u_{2}\right)\right\|_{0,2}^{2} .
\end{aligned}
$$

Hence, with $\epsilon$ small enough

$$
\left\|A\left(v_{1}\right)-A\left(v_{2}\right)\right\|_{k, 2} \leqq \alpha\left\|v_{1}-v_{2}\right\|_{k, 2}, \quad 0 \leqq \alpha<1 .
$$

If we introduce into the set $\|v\|_{k, p} \leqq 2 R, v-u_{0} \in \dot{W}_{p}^{(k)}(\Omega)$, the metric induced by the norm $\|v\|_{k, 2}$, we obtain a complete metric space and the operator $A$ is a contraction. This implies the existence of a fixed point, which is a solution of $(1.2),(1.3)$ belonging to $W_{p}^{(k)}(\Omega)$. From (1.8), this estimation for $\tau_{0} \leqq \tau<\tau+\epsilon$ with $\epsilon$ small enough follows.

(ii) For $0 \leqq \tau \leqq 1$ and $u \in W_{v}^{(k)}$ the solution of the problem, an estimation (1.8) holds with $c(p)$ independent of $\tau$. Let us suppose the contrary. Then for $n$ integers, there exists $\tau_{n}$ and $f_{i}^{n} \in L_{p}, u_{0}^{n} \in W_{p}^{(k)}$, with $u_{n} \in W_{p}^{(k)}$ the solutions of the problem, such that

$$
\left\|u_{n}\right\|_{k, p} \geqq n\left(1+\sum_{|i| \leq k}\left\|f_{i}^{n}\right\|_{0, p}+\left\|u_{0}^{n}\right\|_{k, p}\right) .
$$

Let

$$
t_{n}=\left\|u_{n}\right\|_{k, p}, \quad v_{n}=u_{n} / t_{n} .
$$

If we put $g_{i}^{u}=\left(1 / t_{n}\right) f_{i}^{n}$ and $v_{0}^{n}=u_{0}^{n} / t_{n}$, we obtain that $g_{i}^{n} \rightarrow 0$ in $L_{p}$ and $v_{0}^{n} \rightarrow 0$ in $W_{p}^{(k)}$. We have for $\varphi \in \stackrel{\circ}{W}_{p^{\prime}}^{(k)}$ : 


$$
\begin{aligned}
\left(1-\tau_{n}\right) \int_{\Omega|i|,|j| \leqslant k} & a_{i j}(x) D^{i} \varphi D^{j_{v_{n}}} d x+\tau_{n} \int_{\Omega} \sum_{|i| \leqslant k} \frac{1}{t_{n}} a_{i}\left(x, t_{n} \xi\left(v_{n}\right)\right) D^{i} \varphi d x \\
= & \int \sum_{\Omega|i| \leqslant k} D^{i} \varphi g_{i}^{n} d x \\
= & \int_{\Omega|i|,|j| \leq k} \sum_{i j}(x) D^{i} \varphi D^{j_{v_{n}}} d x \\
& \quad+\tau_{n} \int_{\Omega|i| \leqslant k} \sum_{t_{n}}\left(\frac{1}{t_{i}} a_{i}\left(x, t_{n} \xi\left(v_{n}\right)\right)-\sum_{|j| \leq k} a_{i j}(x) D^{j_{v_{n}}}\right) D^{i} \varphi d x .
\end{aligned}
$$

In virtue of Lemma 2 , we can choose $\varphi_{n}$ such that $\left\|\varphi_{n}\right\|_{k, p^{\prime}}=1$ and

$$
\int_{\Omega|i|,|j| \leq k} a_{i j}(x) D^{i} \varphi_{n} D^{j}\left(v_{n}-v_{0}^{n}\right) d x \geqq c_{1}>0 \quad \text { for } n \geqq n_{0},
$$

which implies for $n \geqq n_{0}^{\prime}$ :

$$
\int_{\Omega|i|,|j| \leq k} a_{i j}(x) D^{i} \varphi_{n} D^{j_{v_{n}}} d x \geqq c_{2}>0 .
$$

Because of (1.7), we obtain

$$
\begin{aligned}
\lim _{n \rightarrow \infty} \mid \int_{\Omega} \sum_{|i| \leqq k}\left(\frac{1}{t_{n}} a_{i}\left(x, t_{n} \xi\left(v_{n}\right)\right)-\right. & \left.\sum_{|j| \leqq k} a_{i j}(x) D^{j} v_{n}\right) \cdot D^{i} \varphi_{n} d x \mid \\
& \leqq c\left(t_{n}\right)\left(\left.\left\|\varphi_{n}\right\|\right|_{k, 1}+\left\|\varphi_{n}\right\|\left\|_{k, p^{\prime}}\right\| v_{n} \|_{k, p}\right) \rightarrow 0
\end{aligned}
$$

which gives, together with (2.5) and because $g_{i}^{n} \rightarrow 0$ in $L_{p}$, the contradiction.

(iii) By standard argument, the set $S$ of $\tau$ where the theorem is valid, is closed; this follows from the fact that if $\tau_{n} \in S$ and $u_{n}$ are solutions, then as above, $u_{n} \rightarrow u$ in $W_{2}^{(k)}$ where $u$ is the solution for $\tau=\lim _{n \rightarrow \infty} \tau_{n}$. But, since

$$
\left\|u_{n}\right\|_{k, p} \leqq c\left(1+\sum_{|i| \leqq k}\left\|f_{i}\right\|_{0, p}+\left\|u_{0}\right\|_{k, p}\right),
$$

the same is true for $u$. As in (ii), the set $S$ is open; so it is the whole interval $\langle 0,1\rangle$. q.e.d.

\section{BIBLIOGRAPHY}

1. F. E. Browder, Existence and uniqueness theorems for solutions of non-linear boundary value problems, Proc. Sympos. Appl. Math., vol. 17, Amer. Math. Soc., Providence, R. I., 1965, pp. 24-49. MR 33 \#6092.

2. E. Giusti, Regolaritd parziale delle soluzioni di sistemi ellitici quasi-lineari di ordine arbitrario, Ann. Scuola Norm. Sup. Pisa (3) 23 (1969), 115-141. MR 40 \#527. 
3. E. Giusti and M. Miranda, Sulla regolaritd delle soluzioni deboli di una classe di sistemi ellittici quasi-lineari, Arch. Rational Mech. Anal. 31 (1968/69), 173-184. MR 38 \#3574.

4. - Un esempio di soluzioni discontinue per un problema di minimo relativo ad un integrale regolare del calcolo delle variazioni, Boll. Un. Mat. Ital. (4) 1 (1968), 219-226. MR 38 \#591.

5. O. A. Ladyženskaja and N. N. Ural'ceva, Linear and quasi-linear equations of elliptic type, "Nauka", Moscow, 1964; English transl., Academic Press, New York, 1968. MR 35 \#1955.

6. J.-L. Lions and E. Magenes, Problemi ai limiti non omogenei. III, V, Ann. Scuola Norm. Sup. Pisa (3) 15 (1961), 41-103; ibid. (3) 16 (1962), 1-44. MR 26 \#4048; 4049.

7. Ch. B. Morrey, Jr., Multiple integrals in the calculus of variations, Die Grundlehren der math. Wissenschaften, Band 130, Springer-Verlag, New York, 1966. MR $34 \# 2380$.

8. - Partial regularity results for non-linear elliptic systems, J. Math. Mech. 17 (1967/68), 649-670. MR 38 \#6224.

9. J. Nečas, Sur la régularitê des solutions faibles des équations elliptiques non linéarires, Comment. Math. Univ. Carolinae 9 (1968), 365-413. MR 39 \#3142.

University of Illinois, Chicago, Illinois 60680 